ESJ Natural/Life/Medical Sciences

\title{
Pratiques Endogènes De La Culture Des Mangues Locales (Mangifera Indica L, Anacardiaceae) Dans Les Départements Du Noun Et De La Lékié Au Cameroun
}

\author{
Christelle Flavie Mbieji Kemayou \\ Chercheur, Institut de Recherche Agricole pour le Développement (IRAD), \\ Station Polyvalente de Foumbot, Cameroun \\ Département de Biologie Végétale, Faculté des Sciences, Université de \\ Dschang, Cameroun \\ Grâce Annih Mbong \\ Maître de conférences, Département de Biologie Végétale, Faculté des \\ Sciences, Université de Dschang, Cameroun \\ Marie Caroline Momo Solefack \\ Maître de conférences, Département de Biologie Végétale, Faculté des \\ Sciences, Université de Dschang, Cameroun

\section{Jean Kuate} \\ $\mathrm{PhD}$, Chercheur, Institut de Recherche Agricole pour le Développement \\ (IRAD), Direction Générale, Yaoundé, Cameroun
}

Doi:10.19044/esj.2021.v17n29p128

Submitted: 29 June 2021

Accepted: 03 August 2021

Published: 31 August 2021
Copyright 2021 Author(s)

Under Creative Commons BY-NC-ND 4.0 OPEN ACCESS

Cite As:

Mbieji Kemayou C.F., Mbong G.A., Momo Solefack M.C. \& Kuate J. (2021). Pratiques Endogènes De La Culture Des Mangues Locales (Mangifera Indica L, Anacardiaceae) Dans Les Départements Du Noun Et De La Lékié Au Cameroun. European Scientific Journal, ESJ, 17(29), 128.

https://doi.org/10.19044/esj.2021.v17n29p128

\section{Résumé}

La présente étude s’inscrit dans le cadre de la valorisation des mangues locales et de la perception endogène des pratiques culturales associées. Cette étude a été menée entre mars et juin 2020, dans les Départements du Noun et de la Lékié, dans onze (11) villages à fort potentiel de manguiers. L’enquête s'est faite par administration des questionnaires et par des observations directes auprès de 300 producteurs de mangues locales. Il en ressort que les producteurs sont en majorité des hommes (82,66\%) mariés, de plus de 50 ans (59,3\%), de niveau primaire (60\%) pour le Noun et secondaire $(59,3 \%)$ pour 
la Lékié. Les surfaces exploitées sont de moins de 1000 m2, localisées au bord de leurs concessions. Dans les deux Départements, l'itinéraire technique telle que recommandée par les experts n'est pas respecté et les techniques de récolte pratiquées sont inappropriées. Elles se font soit par la grimpe, la secousse de l'arbre ou l'utilisation des crochets. Les fruits récoltés sont mal conditionnés et vendus dans les différents contenants. L'anthracnose (Colletotrichum gloeosporiodes) et la chute de fruits immatures sévissent dans les vergers. Huit variétés locales ont été identifiées. Pour une bonne production des mangues locales dans le Noun et la Lékié il est important pour les producteurs d'améliorer leur capacité, d'utiliser les variétés locales greffées et de se former en techniques de conservation des mangues.

Mots clés: Mangue Locale, Enquête, Itinéraire Technique, Système De Production, Cameroun

\section{Endogenous Practices Of Local Mango (Mangifera Indica L, Anacardiaceae) Cultivation In The Noun And Lékié Divisions In Cameroun}

\section{Christelle Flavie Mbieji Kemayou}

Chercheur, Institut de Recherche Agricole pour le Développement (IRAD),

Station Polyvalente de Foumbot, Cameroun

Département de Biologie Végétale, Faculté des Sciences, Université de

Dschang, Cameroun

Grâce Annih Mbong

Maître de conférences, Département de Biologie Végétale, Faculté des

Sciences, Université de Dschang, Cameroun

\section{Marie Caroline Momo Solefack}

Maître de conférences, Département de Biologie Végétale, Faculté des

Sciences, Université de Dschang, Cameroun

\section{Jean Kuate}

$\mathrm{PhD}$, Chercheur, Institut de Recherche Agricole pour le Développement (IRAD), Direction Générale, Yaoundé, Cameroun

\section{Abstract}

This study is part of the valorisation of local mangoes and the endogenous perception of associated cultivation practices. This study was carried out between March and June 2020, in the Noun and Lékié Divisions, in eleven (11) villages with high potential for mango trees. The survey was carried out by administering questionnaires and by direct observations with 300 local mango producers. It appears that the majority of producers are 
married men (82.66\%), over 50 (59.3\%), primary (60\%) for the Noun and secondary (59.3\%) for the Lékié. The areas exploited are less than $1000 \mathrm{~m} 2$, located at the edge of their concessions. In both Divisions, the technical itinerary as recommended by the experts is not followed and the harvesting techniques practiced are inappropriate. They are done either by climbing, shaking the tree or using the hooks. The harvested fruits are poorly packaged and sold in the various containers. Anthracnose (Colletotrichum gloeosporiodes) and immature fruit drop are rampant in orchards. Eight local varieties have been identified. For a good production of local mangoes in Noun and Lékié it is important for producers to improve their capacity, to use grafted local varieties and to be trained in mango conservation techniques.

Keywords: Local Mango, Survey, Technical Itinerary, Production Systems, Cameroon

\section{Introduction}

La mangue est le septième fruit le plus cultivé dans le monde. Appréciée pour ses qualités gustatives, la mangue est une ressource alimentaire intéressante dont les qualités énergétiques, aromatiques et nutritionnelles (Provitamine A, Vitamine C et Potassium) sont reconnues (Brustel, 2018). C'est un arbre fruitier de la famille des Anacardiacées, originaire d'une vaste zone allant du Nord-Est de l'Inde jusqu'aux Philippines. Son fruit, la mangue, est à mésocarpe charnu contenant un seul noyau central. (Kosterman et Bompard, 1993).

Le Cameroun, regorge un potentiel exceptionnel en matière de diversité variétale de mangues locales. C'est sans doute pour cela que le gouvernement en a décidé de faire un pays exportateur au niveau du marché Européen à l'horizon 2025 (Mballa, 2015). Les demandes sont sans cesse croissantes au niveau des marchés locaux et sous régionaux (Gabon, Guinée Equatoriale et Congo).

Certains producteurs avisés et déçus de la chute drastique des prix du cacao (Theobroma cacao L.) et du café (Coffea Sp), ont reconverti leur plantation en verger de manguiers (Mballa, 2015). Cependant, beaucoup reste à faire particulièrement dans le Noun et la Lékié qui sont deux grands bassins de production, où la culture des mangues reste rudimentaire, réduite au verger de case et conduite sans respect de l'itinéraire technique (Rey et al., 2004).

Ce système de culture conduit malheureusement à des pertes importantes avant, pendant et après la récolte. En vue d'améliorer ce système de culture, il apparait important d'évaluer les perceptions paysannes en matière de culture de la mangue locale afin de déterminer les variétés locales existantes, les pratiques paysannes et les différents itinéraires techniques de production et de récolte. L'amélioration du système de culture des mangues 
locales est sans conteste une voie pour accroître les performances de cette importante filière et de réduire la pauvreté en milieu rural.

\section{Matériel et méthodes}

\section{Description de la zone d'étude}

Cette étude s'est réalisée dans le Département du Noun et celui de la Lékié, deux des grands bassins de production de mangues locales. Le Département du Noun qui a pour Chef-lieu Foumban est situé à l'Ouest du Cameroun (Figure 1) dans la zone agro écologique des hauts plateaux. Il s'étend sur une superficie de $7687 \mathrm{~km}^{2}$, il couvre $55.35 \%$ de la superficie totale de la région de l'Ouest (Boukar, 2015). Les coordonnées géographiques sont 5¹8'15'’ de latitude Nord et 10³5’04'’ de Longitude Est. Son climat de type tropical Soudano - Guinéen est influencé par des vents dominants qui soufflent et amènent l'humidité de l'océan. Son altitude est comprise entre 1000 et $1600 \mathrm{~m}$. La température moyenne annuelle est comprise entre 20 et $22^{\circ} \mathrm{C}$, et la pluviométrie varie entre 1500 et $2200 \mathrm{~mm}$ (Boukar, 2015).

Le Département de la Lékié est situé dans la région du Centre (Figure 1) dans la zone forestière humide à pluviométrie bimodale, avec une pluviométrie comprise entre 1500 et $2000 \mathrm{~mm} / \mathrm{an}$. Les coordonnées géographiques sont $4^{\circ} 12^{\prime} 0^{\prime \prime}$ de latitude Nord et $11^{\circ} 24^{\prime} 0^{\prime \prime}$ ' de longitude Est. Il à une altitude comprise entre 500 et $600 \mathrm{~m}$, les sols sont ferralitiques (rouge et jaune), le climat est de type équatorial. Les températures sont comprises entre 19 et $28^{\circ} \mathrm{C}$ (Pompidou, 2000).

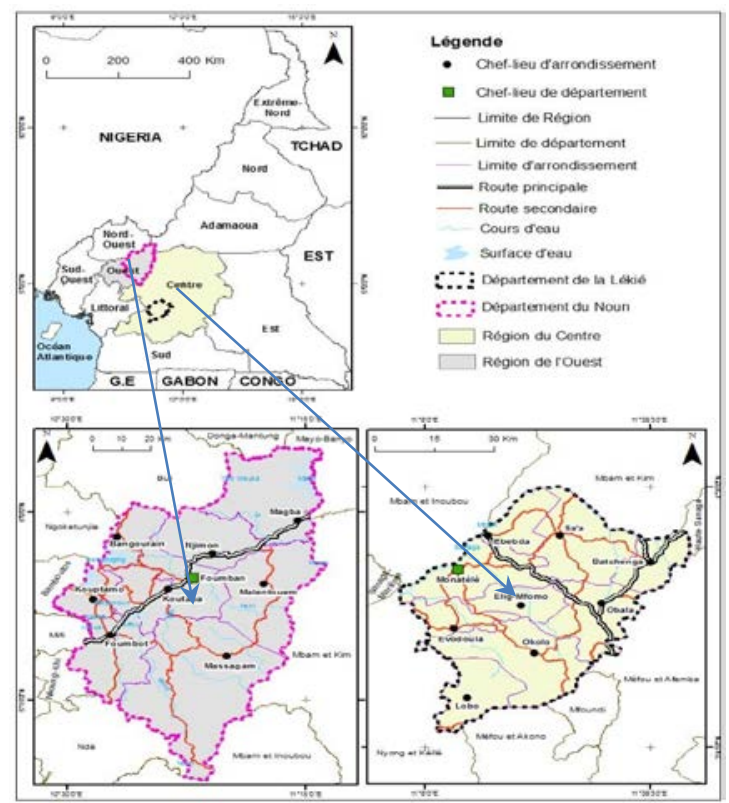

Figure 1. Localisation de la zone d'étude 


\section{Collecte des données}

L'enquête s'est déroulée entre mars et juin 2020, auprès de 300 propriétaires (soit 150 enquêtés par département) d'au moins deux pieds de manguiers en production. L'interview standardisé ou entretien structuré (l'entretien structuré utilise des questions prédéfinies, qui sont posées à tous les enquêtés) a été utilisé au moyen des questionnaires. Cette méthodologie permet un plus grand contrôle des réponses reçues. Cette approche quantitative permet de faire facilement un check-up sur les informations reçues auprès de l'enquêté ou entre les informateurs. En effet, les questions ont porté sur le profil du producteur, l'itinéraire technique, le système de production, les variétés locales existantes produites, les méthodes de vente et les potentiels ennemis de la culture. L'enquête s'est faite dans cinq villages de la Lékié et six villages du Noun, Ces localités représentent les principales zones de production de mangues.

\section{Analyse des données}

La conception du masque et la saisie des données a été faite avec le logiciel Epi Infos 7. Les résultats présentés sous forme de fréquence ont été obtenus suite à une analyse descriptive effectuée à l'aide du logiciel IBM SPSS 22, et représentés sous forme de graphique par le tableur Microsoft Excel 2013.

\section{Résultats}

\section{Caractéristiques sociodémographiques des producteurs}

Les informations reçues auprès des 300 producteurs de mangues dans les deux Départements montrent que dans le Noun et la Lékié, les hommes sont les principaux propriétaires avec une proportion estimée à $82.66 \%$ de la population enquêtées.(Figure 2). Dans le Département du Noun les producteurs appartiennent à deux obédiences religieuses à savoir les religions musulmane (76\%) et chrétienne (24\%). Par contre dans la Lékié, quatre appartenances ont été répertoriées à savoir: les chrétiens (70\%), les animistes (28\%), les musulmans $(0.7 \%)$ et d'autres $(1,3 \%)$. Il s'agit des producteurs témoins de Jéhovah et ceux qui ne pratiquent aucune religion et ou croyance. Très peu de producteurs ont moins de 30 ans (3,3\%) dans le Noun.

Cependant $82,7 \%$ des producteurs enquêtés ont plus de 50 ans et 17,3\% ont une classe d'âge comprise entre 30 et 50 ans. Dans la Lékié, 82,7\% des producteurs enquêtés ont plus de 50 ans et 17,3\% de ceux-ci ont entre 30 et 50 ans. En outre concernant la situation matrimoniale, dans le Noun, 91,3\% des populations interrogées sont mariés indépendamment du type de mariage, très peu sont célibataires (1,3\%), divorcés (2\%) et veufs (ves) (5,3\%). Par contre dans la Lékié , les répondants sont mariés (56,7\%), veufs(ves) (40,7\%) et très peu sont divorcés $(0,7 \%)$ ou célibataires $(2 \%)$. 
L’analyse de la figure 4 nous montre que du côté alphabétisation, dans le Noun $60 \%$ des producteurs ont un niveau primaire, $17,3 \%$ ont un niveau secondaire, $13,3 \%$ ont un niveau supérieur, $8 \%$ n'ont aucun niveau et le reste ont une alphabétisation fonctionnelle comme l'école coranique (Tableau 1). Dans la Lékié, 59 ,3\% ont un niveau secondaire, 36,7\% ont un niveau primaire et1,3\% ont un niveau supérieur.

L’activité principale dans la Lékié est l'agriculture (64\%), ensuite vient le commerce (26,7\%), les salariés (4\%), l'arboriculture fruitière (1,3\%) et enfin les autres activités (2,7\%) comme la maçonnerie, la menuiserie et cordonnerie. Dans le Noun, l'activité principale est également l'agriculture $(41,3 \%)$ suivie des salariées (28,7\%), le commerce (22\%) et l'artisanat (8\%).

Comme activités secondaires dans le Noun, il y'a le commerce (43,3\%), l'agriculture (54\%), l'élevage (1,3\%), l'arboriculture fruitière (1,3\%). Dans la Lékié le commerce est le plus pratiqué (62,7\%) ensuite vient l'agriculture (34\%), les salariés $(0,7 \%)$ l'élevage $(0,7 \%)$, et les autres (2\%).

Pour les producteurs de mangue dans le Noun, leurs principales sources de revenus proviennent des activités agricoles pour $41,3 \%$ et $58 \%$ des activités extra agricoles. Dans la Lékié, 90\% de ceux-ci ont des revenus provenant de l'agriculture et $10 \%$ viennent des activités extra agricoles. Toutes les 300 personnes enquêtées n'appartiennent pas à des GIC (Groupement d'Initiative Commune), et ne bénéficient pas d'un projet et ne sont encadrées par aucun service technique.

Tableau 1. Caractéristiques sociodémographiques des enquêtés

\begin{tabular}{|c|c|c|c|c|}
\hline Caractéristiques & Département & Catégories & Nombre & $\begin{array}{c}\text { Pourcentage } \\
(\%)\end{array}$ \\
\hline \multirow[t]{4}{*}{ Genre } & \multirow[t]{2}{*}{ Noun } & Masculin & 123 & 82,0 \\
\hline & & Féminin & 27 & 18,0 \\
\hline & \multirow[t]{2}{*}{ Lékié } & Masculin & 122 & 81,3 \\
\hline & & Féminin & 28 & 18,7 \\
\hline \multirow[t]{6}{*}{ Obédience religieuse } & \multirow[t]{2}{*}{ Noun } & Musulman & 114 & 76.0 \\
\hline & & Chrétien & 36 & 24.0 \\
\hline & \multirow[t]{4}{*}{ Lékié } & Musulman & 1 & 0,7 \\
\hline & & Chrétien & 105 & 70,0 \\
\hline & & Animistes & 42 & 28,0 \\
\hline & & Autres & 2 & 1,3 \\
\hline \multirow[t]{8}{*}{ Statut matrimonial } & \multirow[t]{4}{*}{ Noun } & Marié & 137 & 91,3 \\
\hline & & Célibataire & 2 & 1,3 \\
\hline & & Divorcé & 3 & 2,0 \\
\hline & & Veuf & 8 & 5,3 \\
\hline & \multirow[t]{4}{*}{ Lékié } & Marié & 85 & 56,7 \\
\hline & & Célibataire & 3 & 2,0 \\
\hline & & Divorcé & 1 & 0,7 \\
\hline & & Veuf & 61 & 40,7 \\
\hline
\end{tabular}




\begin{tabular}{|l|c|c|c|c|}
\hline Niveau d'instruction & \multirow{3}{*}{ Noun } & Primaire & 90 & 60,0 \\
\cline { 3 - 5 } & Secondaire & 26 & 17,3 \\
\cline { 3 - 5 } & Supérieur & 20 & 13,3 \\
\hline \multirow{4}{*}{} & Coranique & 2 & 1,3 \\
\cline { 3 - 5 } & Aucun & 12 & 8,0 \\
\cline { 3 - 5 } & \multirow{4}{*}{ Lékié } & Primaire & 55 & 36,7 \\
\cline { 3 - 5 } & Secondaire & 89 & 59,3 \\
\hline & Supérieur & 6 & 4.0 \\
\hline
\end{tabular}

\section{Méthode d'acquisition de la parcelle}

Dans le Département du Noun, les modes d'acquisition des parcelles sont l'achat sans titre foncier (45,3\%), l'attribution coutumière (22\%), l'achat avec titre foncier (15,3\%), domaine appartenant à l'Etat (11,3\%). C'est généralement les fonctionnaires qui habitent les logements de l’Etat ou les espaces autours de certains services publics, et enfin $2 \%$ des terrains sont acquis par d'autres moyens comme des legs. Dans la Lékié, 60,7\% des producteurs ont acquis les parcelles par attribution coutumière, 37,3 \% des parcelles sont issues des biens de l'Etat et $2 \%$ sont acquises par d'autres moyens (figure 2).

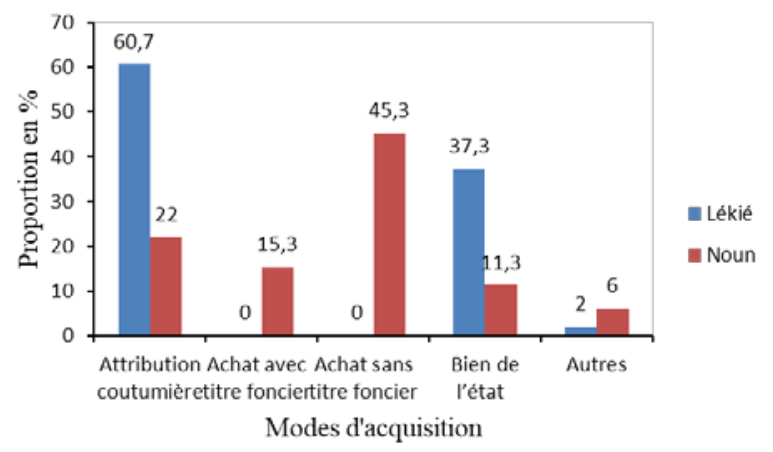

Figure 2. Modes d'acquisition des parcelles en fonction des localités

\section{Système de production des manguiers}

Dans le Noun, les vergers sont situés beaucoup plus dans les concessions familiales (64\%), dans les champs (28\%), dans d'autres lieux (6\%) comme les places publiques et au bord de la route ou des rues (2\%). Dans la Lékié les vergers de manguiers se retrouvent pour la majorité dans les concessions familiales (81,3\%), dans les champs (14\%), au bord de la route $(3,3 \%)$ et dans d'autres endroits $(1,3 \%)$.

Les parcelles exploitées par les populations pour cette culture de mangues sont généralement de petites surfaces de moins de $1000 \mathrm{~m}^{2}$ pour $70 \%$ des enquêtés dans le Noun et $80 \%$ dans la Lékié. Cependant $12 \%$ des producteurs dans la Lékié et $8 \%$ dans le Noun exploitent des superficies allant 
de $1000 \mathrm{~m}^{2}$ à $1 / 2$ ha. Il est remarqué également qu'uniquement dans le Noun $12,7 \%$ des enquêtés exploitent des champs ayant une superficie comprise entre $1 / 2$ ha et 1 ha et $9,3 \%$ exploitent les superficies de plus d'un hectare.

Le relief des parcelles où se trouvent les manguiers dans le Noun est constitué des plaines (86,7\%), des collines (11,3\%), les bas-fonds $(1,3 \%)$ et les versants (0,7\%). Dans la Lékié, le relief des parcelles est beaucoup plus les plaines $(91,3 \%)$ et on retrouve très peu les manguiers plantés sur les collines $(4,7 \%)$, les bas-fonds (2,7\%) et les versants $(1,3 \%)$. Les sols dans le Noun et particulièrement dans les villages ciblés sont latéritiques (82\%), sableux (14\%), argileux (2.7\%), et gravillonnaires (1,3\%). Dans la Lékié les sols des vergers sont latéritiques (34,7\%), sableux (33,3\%) et gravillonnaires (32\%).

L'âge des exploitations de mangue varie entre 3 et 50 ans. Dans le Noun, 44\% des plantations sont âgées entre 25 et 50 ans, 20,7\% des arbres ont entre 15 et 25 ans, $15,3 \%$ ont plus de 50 ans, $18 \%$ ont entre 5 et 15 ans et 2\% des plantations ont entre 3 et 5 ans. Dans la Lékié, 38 ,7\% des plantations ont entre 25 et 50 ans, $28 \%$ des plantations ont entre 15 et 25 ans, $24 \%$ ont plus de 50 ans, 8,7\% ont entre 5 et 15 ans et $0,7 \%$ ont entre 3 et 5 ans (figure $3)$.

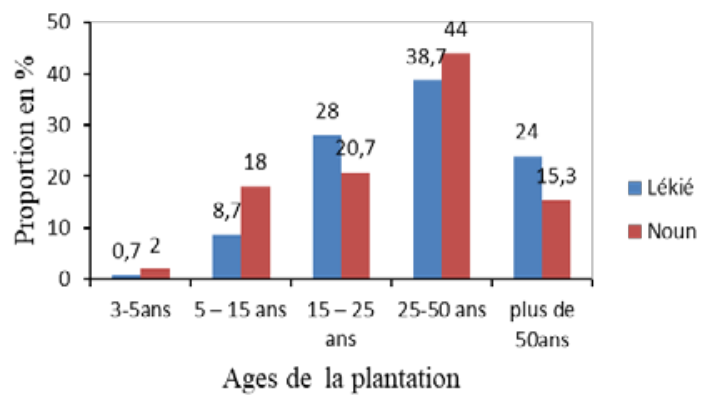

Figure 3. Age des plantations en fonction des localités

\section{Itinéraires techniques}

L'itinéraire technique pour la mise en place des manguiers diffère d'un producteur à un autre, la distance de plantation $10 \mathrm{~m} \mathrm{x} 10 \mathrm{~m}$ telle que recommandée par les experts est respecté par $14 \%$ des producteurs dans la Lékié et 14,7\% dans le Noun. Cependant plusieurs ne tiennent pas compte des dimensions des écarts, ainsi observe-t-on des écartements épars d'un pied à un autre avec des dimensions variant entre 1 et $5 \mathrm{~m}$. Il s'agit de $46 \%$ des enquêtés dans le Noun et 33,3\% dans la Lékié. Une autre partie des producteurs soit 22,7\% dans le Noun et $45,3 \%$ dans la Lékié préfèrent les dimensions $8 \mathrm{mx} 8 \mathrm{~m}$ et une minorité ont des espacements de $6 \mathrm{mx} 6 \mathrm{~m}$ ceux-ci représente respectivement7,3\% et 16,7\% des producteurs de la Lékié et du Noun (Figure 4). 


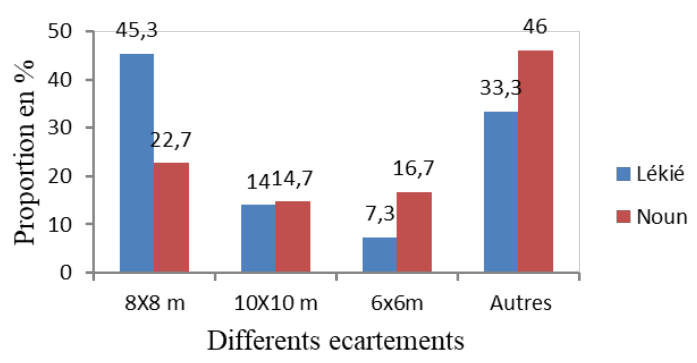

Figure 4. Ecartement entre plants en fonction des localités

La plupart des arbres ne sont pas alignés ce qui traduit un défaut de piquetage, il s'agit de $74,7 \%$ dans le Noun et $87,3 \%$ dans la Lékié et d'autres manguiers sont disposés en ligne ou en rangées. Il s'agit de $12,7 \%$ des enquêtés dans la Lékié et 24,7\% dans le Noun. Les producteurs n'utilisent pas de matériel (motopompe, arrosoirs, pulvérisateur et autres), n'arrosent pas leurs plants, n’utilisent aucun intrant agricole et les différents vergers visités n’ont pas de clôture. Contre les incendies dans la Lékié, 94\% des producteurs ne font aucune pratique pour prévenir des feux de brousse et $6 \%$ seulement des producteurs font des cultures intercalaires pour lutter contre les mauvaises herbes. Dans le Noun 36\% des populations font des cultures intercalaires et 64 $\%$ ne font aucune pratique.

\section{Variétés, méthode de mûrissement et ravageurs}

Dans le Noun quatre variétés (04) locales ont été identifiées à savoir: les Mangues Dame ou Améliorée du Cameroun, les Mangues Bamouns, les Mangues Américaine et les Mangues Allemande. Dans la Lékié sept variétés de mangues ont été répertoriées, il s’agit des Mangues Dames, Koussa Dog, Mangues papayes, Dog Beti, Dshang Dshang 1, Dshang Dshang 2 et Mangues Garoua (Figure 5).

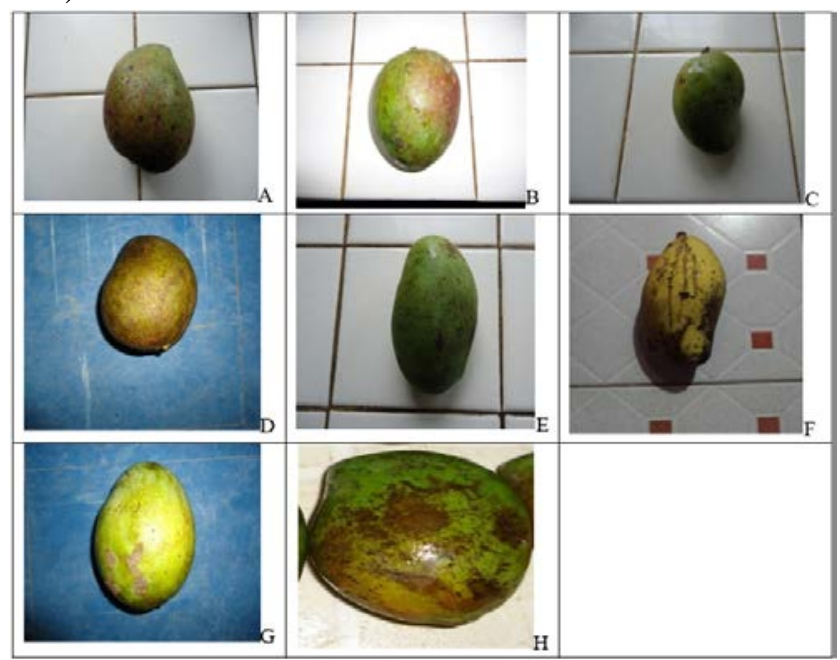


Figure 5 : Photo illustrant les variétésde mangues rencontrées dans les deux Départements : Mangue Allemande ou Mangue Papaye (A), Mangue Americaine (B), Mangue Bamoun ou Dog Beti (C), Mangue Garoua (D), Ameliorée du Cameroun (E), Dshang Dshang 2 (F), Kousa dog (G), Dshang dshang 2 (H).

Le mode méthode de mûrissement est le mûrissement sur l'arbre, dont l'élément indicateur est l'évolution de la coloration de l'épiderme. Cependant les paysans font face à certaines maladies et ravageurs qui accroissent les pertes post récolte comme l'anthracnose (Colletoricum gloeosporiodes) sur feuille. Cette maladie fongique touche beaucoup plus les producteurs de la Lékié $(91,3 \%)$ et $37,3 \%$ des populations du Noun. Les mouches de fruits et les cochenilles blanches ont été observées uniquement dans les vergers du Noun en raison de 2 et $8,7 \%$ respectivement. Les autres phénomènes observés ou relevant des plaintes des producteurs comme le dépérissement du manguier et la chute des fruits immatures ont été observés chez $52 \%$ des populations dans le Noun et 8,7 \% dans la Lékié (Figure 6).

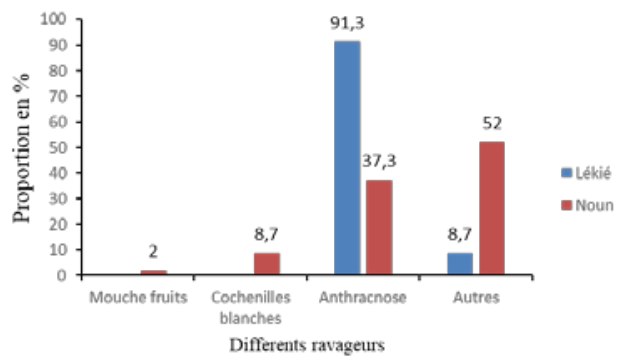

Figure 6. Fréquence des bios agresseurs du manguier dans le Noun et la Lékié

\section{Période de récolte, méthode de récolte, période de vente et type de conservation}

La période de récolte pour plusieurs variétés de mangues se situe au mois de mars dans la Lékié (86,7\%) et dans le Noun (71,3\%). Les variétés précoces commencent à produire en Janvier dans la Lékié et Février dans le Noun. Il s'agit des mangues Bamouns ou Dog Beti (Tableau 2). Les mangues sont récoltées principalement avec les crochets. Ceci concerne 85,3\% des producteurs dans la Lékié et $65,3 \%$ dans le Noun. D’autres moyens comme la grimpe et la lance des projectiles sont également employés pour récolter les mangues pour 14,7\% des populations dans la Lékié et 34\% de celle du Noun (Figure 7). Les méthodes de vente diffèrent d'une zone à une autre. Dans la Lékié les producteurs vendent leurs fruits beaucoup plus en cageots (72,7\%) et en tas (27,3\%). Dans le Noun, la méthode de vente en contenant varié est la plus utilisée $(44,7 \%)$, d'autres méthodes telles que les sacs ou les caisses (27,3\%), en tas $(19,3 \%)$ et en cageots $(8,7 \%)$ sont également employées (Figure 8). Les fruits invendus qui se détériorent avec le temps sont généralement jetés $(98,6 \%)$ et parfois très peu de personnes les transforment 
en fumure. Il concerne 1,4\% des producteurs dans la Lékié et $0,7 \%$ dans le Noun. Aucune méthode de conservation n'est employée par les producteurs. Cependant dans le Noun, il existe une usine de séchage de mangues pour l'export, et une unité de transformation de mangues en confiture chez les moines de Koutaba. Ces différentes unités de transformation s'approvisionnent en tant que commerçants grossistes chez les producteurs. La vente des fruits a une incidence financière dans le porte-monnaie des producteurs, $98 \%$ des enquêtés dans la Lékié et 81,3\% dans la Noun affirment que les revenus ne sont pas assez. $18 \%$ des producteurs du Noun et $2 \%$ de ceux de la Lékié trouvent que les revenus sont moyens et $0,7 \%$ des répondants du Noun avouent que les revenus issus de la vente sont assez consistants. C'est pour la plupart ceux qui ont une grande superficie d'exploitation. L'argent issu des ventes des fruits permet de subvenir aux besoins de première nécessité pour $93,3 \%$ des producteurs dans la Lékié et $77 \%$ dans le Noun. Bien plus, 22\% des producteurs du Noun et 6,7\% de ceux de la Lékié utilisent les revenus issus de la vente des mangues pour investir dans les domaines divers et 3,7\% des producteurs du Noun agrandissent leur exploitation avec leurs revenus (Figure 9).

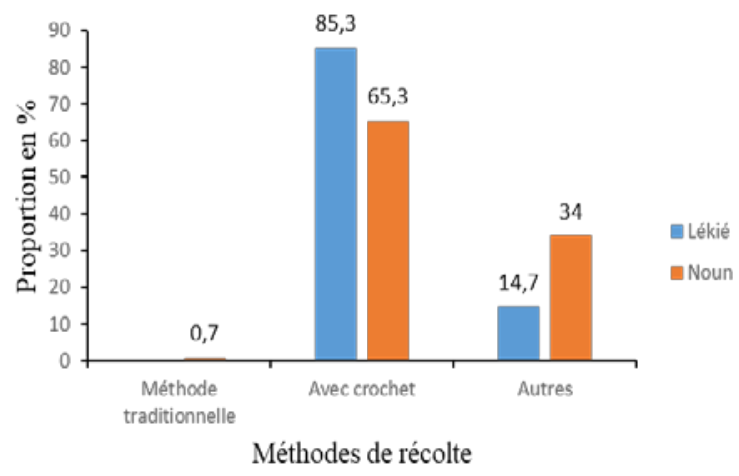

Figure 7. Regroupement en fonction de la méthode de récolte utilisée

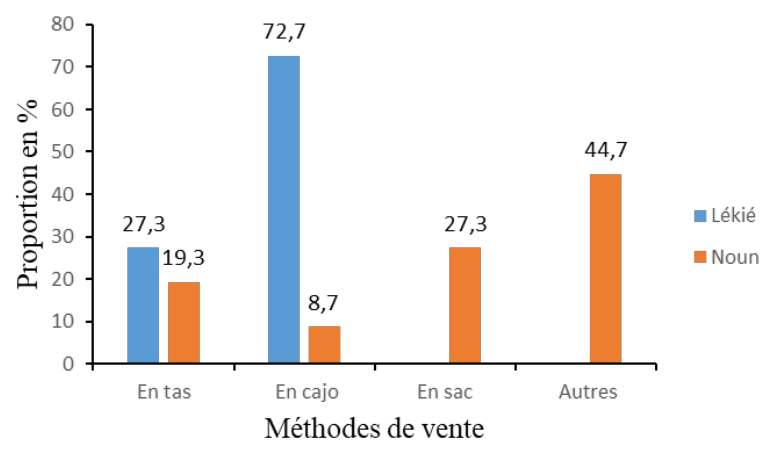

Figure 8. Regroupement des producteurs en fonction des méthodes de conditionnement pour la vente 


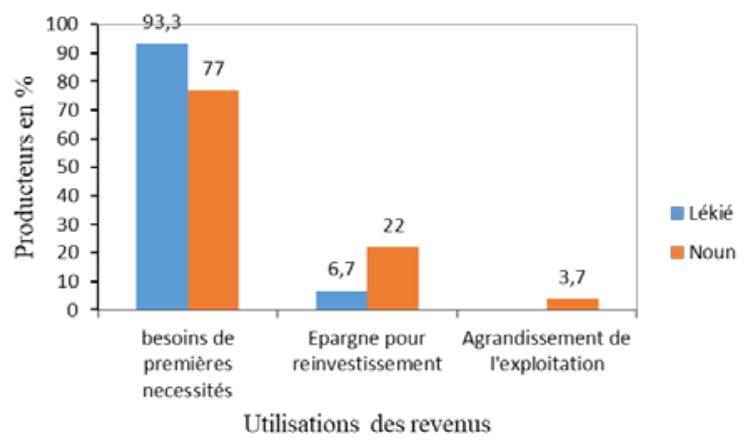

Figure 9. Regroupement des producteurs en fonction de l'utilisation des revenus

Tableau 2. Période de production des mangues locales dans le Noun et la Lékié

\begin{tabular}{|c|c|c|c|c|c|c|c|}
\hline Variétés & Janvier & Février & Mars & Avril & Mai & Juin & Juillet \\
\hline Mangues Améliorée du & & & & & & & \\
\hline $\begin{array}{c}\text { Cameroun } \\
\text { Mangue Américaines } \\
\text { (Noun) ou Mangue papaye } \\
\text { (Lékié) }\end{array}$ & & & & & & & \\
\hline Mangue Allemandes & & & & & & $\Rightarrow$ & \\
\hline $\begin{array}{l}\text { Mangue Bamoun (Noun) } \\
\text { ou Dog Beti (Lékié) }\end{array}$ & 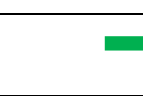 & & $\Rightarrow$ & & & & \\
\hline Dshang Dshang 1 & & & & & & & \\
\hline Dshang Dshang 2 & & & & & & & \\
\hline Kousa Dog & & & & & & & \\
\hline Mangue Garoua & & & & & & & \\
\hline
\end{tabular}

\section{Discussion}

\section{Caractéristiques sociodémographiques des producteurs}

L'enquête a révélé que 82.66 \% des enquêtés sont des hommes et $17.33 \%$ sont des femmes. En effet les femmes s'intéressent beaucoup plus aux travaux ménagers, à l'agriculture dans le domaine des cultures annuelles, semis annuelle et le commerce. Les hommes quant à eux ont beaucoup plus une vision pérenne dans l'agriculture. Les rares femmes propriétaires sont généralement les veuves ou les héritières. Ces résultats sont en parfait accord avec ceux trouvés par Kuaté et al. (2006) ; Mballa, (2015) ; Passannet et al. (2017) où dans leurs différentes études, ont trouvé que moins de 13\% des propriétaires des vergers sont les femmes. La religion dominante dans le Noun pour les villages enquêtés est la religion musulmane avec comme ethnie prédominante les Bamouns, les Bamilékés et les Tikars. Par contre dans la 
Lékié c’est beaucoup plus les chrétiens avec comme ethnie prédominante les Manguissa'a et les Bassa'a pour les zones enquêtées. La grande majorité des producteurs soit $67,3 \%$ ont plus de 50 ans, ceci traduit le fait que cette activité intéresse les personnes assez âgées. En effet , cette situation serait la cause du problème de pérennité de cette culture, étant donné que très peu de jeunes (3.3\%) s'intéressent à cette culture au profit des métiers moins salissants et/ou rentables à court terme comme la moto taxi ou la pratique des cultures maraichères.

Ces résultats sont analogues à ceux trouvés par AFRISTAT (2001) au Mali et Passannet et al. (2017) au Tchad lors d'une enquête test sur l'estimation de la culture fruitière où $56,7 \%$ des enquêtés avaient plus de 50 ans. Les producteurs pour la plupart sont mariés et très peu sont célibataires et où divorcés, ce qui corrobore avec les résultats de Kuaté et al. (2006) dans les zones péri urbaines de Yaoundé au Cameroun où une enquête diagnostic $\mathrm{a}$ été faite sur les exploitations fruitières et où $77 \%$ des enquêtés sont mariés. En outre le niveau d'instruction est primaire dans le Noun (60\%) et secondaire dans la Lekié (59,3\%). Ces résultats sont similaires à ceux trouvés par Kuaté et al. (2006) où $69,4 \%$ des producteurs des fruits ont un niveau primaire. Leur activité principale est l'agriculture, et en second plan le commerce. Il est à noter que cette tendance serait due au fait que l'enquête s'est déroulée dans les zones rurales et où ces deux activités sont courantes. Ces résultats sont en accord avec ceux de Bourou et al. (2019) et Sougnabe et al. (2010).

\section{Aires et relief d'exploitation}

La superficie exploitée est généralement de moins de $1000 \mathrm{~m}^{2}$ car la plupart des arbres se trouvent en bordure des maisons d'habitation des propriétaires et le mode d'acquisition est l'achat sans titre foncier pour le Noun (45,3\%), et l'attribution coutumière $(60,7 \%)$ pour la Lékié. Donc les exploitants sont propriétaires des terres cultivées, ce qui est en accord avec les résultats observés dans la région du Nord Cameroun par Bourou et al. (2019). La majorité des manguiers ont un âge compris entre 25 et 50 ans. Ces arbres sont donc vieillissants et posent un problème de la pérennité de la culture.

Le relief des parcelles où sont plantés les manguiers est pour la majorité des plaines et les sols sont pour la plupart latéritiques ce qui est un atout car les sols ferralitiques ont un effet favorable sur la coloration externe de la mangue et sur leur qualité interne (Vannierre et al, 2004).

\section{Matériels utilisés, type d'arrosage et intrants agricoles}

Les enquêtés affirment également qu'ils n'utilisent aucun matériel particulier pour l'entretien de leurs arbres, car les arbres sont des variétés polyembryonnés âgées qui sont des variétés assez rustiques adaptées au sol et résistants à certaines maladies et ne nécessitant pas des soins particuliers. De 
ce fait, l'arrosage est essentiellement pluvial et en saison sèche les racines assez développées s'alimentent en eaux et éléments minéraux dans la nappe phréatique. Aucun intrant n'est utilisé pour ces plants à part la seule fumure issue des feuilles mortes et des fruits décomposés tombant en dessous de l'arbre. Ces résultats sont similaires à ceux trouvés par Mballa (2015) où 74\% en moyenne des exploitants fruitiers ne font rien pour maintenir la fertilité de leurs champs. Passannet et al.(2017) ont également trouvé qu'au Tchad l'utilisation des intrants chimiques et fumier est peu répandu. Et pourtant, les agronomes conseillent aux producteurs de faire le premier apport en fumure un mois après la plantation, et les années suivantes apporter la moitié de la dose en juin, l'autre en septembre et pendant la nouaison (Normand et Hamasselbe, 1991).

Les observations sur le terrain ont également montré que l'écartement entre les manguiers ne respecte pas un ordre logique. Ils sont épars et varient d'une plantation à une autre. Certains producteurs pratiques des cultures intercalaires comme les légumineuses et céréales. Aucun des vergers de case observés n'a de manguiers alignés. Ceci traduit l'ignorance de l'itinéraire technique. Or un écartement de 10 mètres est recommandé entre les manguiers (Vannierre et al, 2004) pour pouvoir circuler entre les arbres, éviter l'encombrement des frondaisons et éviter l'étiolement causé par le manque de soleil.

\section{Lutte contre les incendies}

Pour lutter contre les incendies, les producteurs du Noun pratiquent des cultures intercalaires pour entretenir les intervalles, ce qui est une pratique à encourager car les cultures intercalaires permettent de protéger le sol contre l'érosion, apportent la matière organique, favorisent la porosité et diminuent le tassement du sol (Vanniere et al, 2004). Par contre, dans la Lékié les producteurs n'utilisent ni herbicide, ni défrichage et culture intercalaire, pour la simple raison qu'aucune culture ne pousse en dessous des manguiers. Cela se justifie par le fait qu'aucune pratique de taille de formation et de croissance n’est pratiquée. Cela induit un couvert fermé, bloquant ainsi la pénétration des rayons lumineux dans les intervalles. Ces résultats sont similaires à ceux trouvés par Passannet et al (2017) où 51\% des producteurs du Tchad ne pratiquent de taille par méconnaissance des bienfaits de cette pratique et aussi par peur des taxes infligées par les agents de protection de l'environnement. Les différents vergers n'ont pas d'enclos particuliers que ce soit avec les haies et fil barbelé, cela est dû à une certaine assurance des propriétaires, car les arbres étant adultes ne sont plus à la portée des animaux domestiques et des bêtes en divagation. 


\section{Variétés, récolte et murissement}

Les accessions locales identifiées sont au nombre de huit. Il faut noter que dans la Lékié certaines variétés n’ont pas pu être décrites à cause de l'absence des fruits sur l'arbre. Certaines accessions sont communes aux deux Départements et diffèrent uniquement par les appellations locales. C'est le cas des Améliorées du Cameroun ou Mangues Dames dans les deux Départements, Dog Béti dans la Lekié et Mangue Bamoun dans le Noun, Mangue Américaine dans le Noun et Mangue Papaye dans la Lekié. Le nombre de varieté obtenue dans cette étude est supérieur à ceux trouvés au Tchad par Passannet et al. (2017) qui ont identifiés quatre variétés locales, et Ternoy et al. (2006) au Sénégal qui ont identifié six variétés locales.

La période de production des mangues est de fin janvier pour certaines variétés précoces qui sont les variétés qui ont la capacité de produire les fruits avant les autres (Rey et al, 2004) comme les Mangues Bamoun dans le Noun, Dog Béti dans la Lékié, et s'étendent jusqu’à juillet pour la variété Améliorée du Cameroun. Ces résultats sont similaires à ceux de Kuaté et al, (2006), dans les zones périurbaines de Yaoundé qui ont trouvé que les mangues vertes produisent entre février et mai. De même au Tchad, Passannet et al. (2017) au cours d'une enquête ont remarqué que les mangues locales commencent à produire de fin janvier et s'étendent jusqu’à début août.

Les récoltes sont programmées lorsqu'il y’a évolution de la coloration de l'épiderme qui passe du vert foncé au vert pâle. La récolte se fait beaucoup plus avec les crochets, la grimpe (méthode traditionnelle), la lance des cailloux, et le ramassage des fruits tombés au sol. Ces différentes pratiques, particulièrement la méthode traditionnelle est la plus pratiquée au Tchad d'après Passannet et al. (2017).

Le mûrissement se fait généralement sur l'arbre et aucune pratique de conservation n'est utilisée. La méthode de vente la plus utilisée est la mise en cageot et en contenant varié quel que soit la zone. Les revenus servent à assurer les besoins de première nécessité. Les fruits avariés sont jetés .Il est à noter que beaucoup de fruits ne sont pas destinés à la vente et servent beaucoup plus de dessert ou d'amuse-gueule. Des observations similaires ont été faites par Kuaté et al. (2006) et Bourou et al. (2019). Les maladies les plus répandues sont beaucoup plus l'anthracnose sur feuille et celles causant la chute des fruits immatures et le dépérissement du manguier. Les résultats pareils ont été trouvés par (Vannierre et al, 2004).

\section{Conclusion}

A l'issue de cette enquête dans le Département du Noun et de la Lékié qui sont deux Départements situées dans deux zones agro écologiques différentes et ayant un fort potentiel de mangues locales, on constate que la culture de la mangue reste rudimentaire et pratiquée par les personnes âgées 
ayant un niveau intellectuel primaire et ne bénéficiant d'aucun encadrement technique. Cependant on note que les pratiques sont nettement meilleures dans le Noun, à cause du marché très développé de ce fruit vers certains pays de la sous-région et la présence d'une grande usine de séchage de mangues à Foumbot. Huit accessions locales ont été répertoriées mais elles sont produites sans respect de l'itinéraire technique tel que recommandé par les spécialistes. Il serait plus intéressant de mettre en valeur ces différentes accessions en les caractérisant morphologiquement et physiquement. Les techniques de récolte sont à améliorer et les méthodes de conservation sont à penser . Malgré la présence de certains ravageurs causant d'énormes pertes, l'activité procure des revenus modestes permettant d'assouvir les besoins de première nécessité. Toutefois, on conseille aux différents producteurs :

- D’agrandir leur surface d'exploitation en créant des véritables vergers sur des surfaces appropriées ;

- De respecter l'itinéraire technique de plantation des arbres fruitiers en se rapprochant des instituts de recherche comme l'IRAD (station de Foumbot dans le Noun et Nkolbisson dans la Lékié) en vue d'un meilleur suivi ;

- Il serait bénéfique pour eux de se regrouper en GIC ou en Coopérative pour trouver des stratégies afin de mieux écouler leur produit ;

- D'étaler leur production en se procurant les variétés locales greffées auprès des instituts de recherche, qui produiront en un temps relativement court (03 ans) et en contre saison.

\section{References:}

1. AFRISTAT, (2001). Rapport final d'enquête test sur l'estimation de la production fruitière. Observatoire Economique et Statistique d’Afrique Subsaharienne. Mali, série méthode numéro 5, 146p.

2. Boukar, O. (2015). Caractéristiques socio-économiques et techniques de l'élevage bovin à viande dans le département du Noun, région de l'ouest Cameroun, mémoire d'ingénieur FASA Dschang, 88p.

3. Bourou, S., Adamou, I., Madou, C., Kuaté, J. (2019). Diagnostic du mode d'exploitation et contraintes majeures des vergers fruitiers et autres espèces arboricoles dans la région du Nord Cameroun. Int. J. Biol. Chem. Sci, 13(1) : 215-264.

DOI :https://dx.doi.org/10.4314/ijbcs.v13i1.21

4. Brustel, L. (2018). Evaluation de l'effet de pratiques culturales (paillage / enherbement du sol / récolte prophylactique précoce) en vergers de manguier (Mangifera indica L.) sur la régulation de bio agresseurs de la floraison et de la fructification : les cas de la cécidomyie des fleurs (Procontarinia mangiferae) et des mouches des 
fruits (Diptera : Tephritidae), mémoire d'ingenieur, INP de Toulouses, p.117.

5. Kosterman, AJ., Bompard, JM. (1993). The mangoes, their botany, nomeclature, horticulture and utilization. Academic Press. London, p. 233.

6. Kuate, J ., Bella, M ., Damesse, F., Kouodiekong, L., Atanga, SN., David, O., Parrot, L. (2006). Enquête sur les cultures fruitières dans les exploitations familiales agricoles en zone humide du Cameroun. Fruits, 61(6): 373-387.

DOI: 10.1051/fruits:2006037 www.edpsciences.org/fruits.

7. Mballa-Mbarga, CY. (2015). Etude diagnostique sur le développement de la culture fruitière dans le Centre, l'Adamaoua et le Nord Cameroun. Rapport de mission de l'organisation des Nations Unies pour l'Alimentation et l'agriculture, p.55.

8. Normand, F., Hamasselbe, A. (1991). Fiche technique manguier. IRFA.CIRAD, N²1.IRA Section fruits, Garoua, p.12.

9. Passannet-Schinzoumka, A., Aghofack-Nguemezi, J., Gatsing, D.(2017). Diversité de variétés, de production et de conservation des mangues au Tchad. Int. J. Biol .Chem. Sci, 11 (3).1145-1164.DOI : https://dx.doi.org /10.4314 /ijbcs.u11i318

10. Pompidou, D. (2000). Analyse économique de l'intégration de l'élevage du poulet de chair dans les systèmes de production agricole de la Lékie : cas de Nkometou III, mémoire de master II, Université de Dschang, p.95.

11. Rey, JY., Diallo, TM., Vannière, H., Didier, C., Kéita, S., Sangaré, M. (2004). La mangue en Afrique de l'Ouest francophone: variétés et composition variétale des vergers. Fruits, 59(3): 191-208. DOI: 10.1051/fruits:2004018.

12. Sougnabe, SP., Woin, N., Lyannaz, J P., Rey, JY., Sali B. (2010). Caractérisation des bassins et des systèmes de production fruitière dans les savanes d'Afrique centrale. Acte du Colloque des Savanes africaines en développement : innover pour durer, Garoua, Cameroun. Cirad, $10 \mathrm{p}$.

13. Ternoy, J., Austin, JE., Poublanc, C., Diop, M., Nugawela, P., Dioh S. (2006). La chaine de valeurs de la mangue au Sénégal. Analyse et cadre stratégique d'initiatives pour la croissance de la filière. Rapport du Programme USAID/Croissance économique, 91p.

14. Vanniere, H., Didier, C., Rey, JY., Diallo, M., Keita, S., Sangaré, M. (2004). La mangue en Afrique de l'ouest francophone : les systèmes de production et les itinéraires techniques. Fruits, vol .59(6) : 383-398. DOI : 10.1051 /fruits : 2005001. 